\title{
Trends in diagnostic techniques and factors associated with tuberculosis treatment outcomes in Lesotho, 2010-2015
}

\author{
Eltony Mugomeria $^{{ }^{*}}$ iD Bisrat S Bekele $^{\mathrm{a}}$ (D), Charles Maibvise ${ }^{\mathrm{b}}$ (D) and Clemence Tariraic iD \\ ${ }^{a}$ Faculty of Health Sciences, Department of Pharmacy, National University of Lesotho, Maseru, Lesotho \\ ${ }^{b}$ Faculty of Health Sciences, Department of Nursing, University of Swaziland, Mbabane, Swaziland \\ 'Department of Pharmaceutical Sciences, Tshwane University of Technology, Pretoria, South Africa \\ *Corresponding author, email: emugomeri@nul.Is
}

Tuberculosis is a global public health problem. Lesotho, a sub-Saharan country with high HIV and tuberculosis burden, launched the Three I's programme of the World Health Organization in 2013. However, the outcomes of this intervention are scarcely known. This study evaluated the profile of tuberculosis diagnostic techniques used, treatment outcomes and the associated factors across the baseline period (2010-2012) and after the launch of the Three l's programme (2013-2015) based on a retrospective cohort review of patient records at a major clinic in Maseru, Lesotho. In total, 812 cases with complete records from 1066 randomly selected cases treated (every second case from the sampling frame) were included in the study. Factor analysis was based on logistic regression analysis. The use of the GeneXpert MTB/RIF technique remained below $20 \%$ throughout the study period. Overall, $28.9 \%(n=812)$ cases had unsuccessful tuberculosis treatment. Male gender $(p=0.046)$, extra-pulmonary tuberculosis $(p=0.002)$ and treatment observation by community health workers $(p=0.001)$ were significantly associated with unsuccessful treatment outcome. Overall, treatment outcomes did not differ significantly $(p=0.636)$ before and after the launch of the intervention. These findings indicate the need to strengthen the implementation of the Three l's programme, taking into account the significant factors in this study.

Keywords: community health workers, extra-pulmonary tuberculosis, GeneXpert MTB/RIF, HIV, Three l's programme

\section{Introduction}

Tuberculosis (TB) is a severe global public health problem. The incidence of TB in 2013 was about 126 cases per 100000 global population and the number of annual deaths associated with TB was about 1.3 million. ${ }^{1}$ Patients co-infected with human immunodeficiency virus (HIV) and tuberculosis (HIV/TB) have an increased risk of treatment failure, relapse and death. ${ }^{2}$ In addition, HIV complicates the diagnosis of TB. ${ }^{3}$

The global strategy to improve TB detection and treatment outcomes remains focused on rapid diagnosis and drug sensitivity testing (DST) of Mycobacterium tuberculosis (MTB) strains. ${ }^{4}$ Chest X-ray and smear microscopy, regarded as less effective diagnostic techniques for TB, remain predominant in sub-Saharan Africa. The use of GeneXpert MTB/RIF (Cepheid, Sunnyvale, CA, USA) and routine culture of TB suspect specimens remain low due to high cost and laboratory infrastructure constraints. ${ }^{5}$ The GeneXpert MTB/RIF test is recommended by the World Health Organization (WHO), particularly for screening TB in HIV co-infected individuals. ${ }^{6}$ However, data on the trends of its use in developing countries remain scarce.

The WHO launched the Three I's programme, comprising intensified case finding (ICF), isoniazid preventive therapy (IPT) and tuberculosis infection control, in 2010 to reduce the incidence of TB, particularly in regions with high TB burden in Africa.' Lesotho, a sub-Saharan developing country, launched this programme in $2013 .{ }^{8}$ The use of GeneXpert MTB/RIF for TB diagnosis and detection of multi-drug resistant TB (MDR-TB) was central to ICF. ${ }^{9}$ The Three l's programme was launched after the Directly Observed Treatment Short-course (DOTS), a WHO programme whose goal was to intensify monitoring of adherence and compliance to TB treatment. ${ }^{7}$ However, the DOTS programme has been criticised for failing to eradicate TB in the worst-affected areas. ${ }^{1}$
Lesotho continues to face many challenges in tackling the problem of TB. The estimated prevalence of TB has remained above 400 cases per 100000 population since 2006.' In 2014, Lesotho had the fourth highest prevalence of TB worldwide.' Dhemba, Mushonga and Mugomeri ${ }^{10}$ note that the millennium development goal (MDG) of scaling up the surveillance and treatment of at least $80 \%$ TB/HIV co-infected people by 2015 was off-target. Laboratory diagnosis for TB is one such major challenge in the country. Late diagnosis of TB remains a major cause of TB treatment failure, death and increased transmission of the disease. ${ }^{11}$ This study therefore reviewed the trends in the use of TB diagnostic techniques, treatment outcomes and identified covariates associated with unsuccessful treatment outcomes across the baseline period (2010-2012) and after the launch of the Three I's programme (2013-2015) in Lesotho.

\section{Study population and methods}

\section{Study setting}

The study was conducted at Senkatana HIV and TB clinic in Maseru District, Lesotho. Senkatana is the largest governmentowned antiretroviral therapy (ART) centre in Maseru. Lesotho is an enclave in South Africa with about two million people. ${ }^{12}$ The country is divided into 10 administrative districts with the capital city, Maseru, in Maseru District. With 40\% of its population living below the official poverty line of US\$1.25 per day, the United Nations $^{13}$ classifies the country as a low-income country. Approximately $23 \%$ of the population is infected with HIV and about $75 \%$ of patients with TB are co-infected with HIV. ${ }^{11}$

\section{Study design, population and sample size}

A retrospective cohort review of the tuberculosis (TB) patients registers at Senkatana HIV and TB clinic in Maseru District, Lesotho was conducted between March and April 2016. 
According to the records, 2132 patients were treated for TB at Senkatana from 2010 to 2015. Data were extracted from the TB patients'registers using a systematic random sampling technique based on a sampling frame. A list of all records available at the data collection site represented the sampling frame. Every second record, in consecutive order, was selected from the sampling frame and was included in the study. Only patients with TB treatment outcomes reported in their records were included in the study. Patients enrolled before January 1, 2010 and after December 31, 2015 and records with missing data on the variables studied were excluded from the study.

\section{Data collection and analysis}

Data comprising demographic, clinical variables, TB treatment regimens and outcomes were extracted using a Microsoft Access $^{\circledR}$ (Microsoft Corp, Redmond, WA, USA) database tool designed by the researchers. Data were collected between March and April 2016. Statistical significance $(p<0.05)$ of the associations between demographic and clinical variables, including HIV status, and treatment outcomes were tested using univariate and multivariate logistic regression in Stata ${ }^{\oplus}$ version 13 software (StataCorp, College Station, TX, USA).
Treatment failure, default, cure and treatment completion were defined according to the DOTS treatment protocol of the WHO.? Overall, unsuccessful treatment referred to treatment failure, default, death or transfer out, while treatment success referred to a positive cure outcome or treatment completion.

\section{Ethical considerations}

The research proposal was approved by the Institutional Ethical Review Board of the Faculty of Health Sciences at the National University of Lesotho and the Ethics Committee of the Ministry of Health of Lesotho. Permission to conduct the study was also granted by the hospital authority. Patient names were not collected and all data were treated with confidentiality.

\section{Results}

Of 1066 reviewed, only 812 (76.2\%) records had data on TB treatment outcomes and were included in the final analysis. The ages of the patients ranged from 1 to 90 years and the median age was 35 (interquartile range 29-44). Table 1 presents patient characteristics categorised by TB treatment outcomes. Males constituted $55.2 \%$ and about $84.7 \%$ were new TB cases, while $83.0 \%$ were HIV-positive. In total, $60.3 \%$ patients had pulmonary

Table 1: Tuberculosis treatment outcomes stratified by demographic characteristics

\begin{tabular}{|c|c|c|c|c|}
\hline \multicolumn{5}{|c|}{ Treatment outcome } \\
\hline Characteristics & Successful & Unsuccessful & Total (\%) & $p$-value \\
\hline \multicolumn{5}{|l|}{ Gender } \\
\hline Male & $298(51.6)$ & $150(64.2)$ & $448(55.2)$ & $<0.001$ \\
\hline Female & $279(48.4)$ & $85(35.8)$ & $364(44.8)$ & \\
\hline Total & $577(100)$ & $235(100)$ & $812(100)$ & \\
\hline \multicolumn{5}{|l|}{ Age (years) } \\
\hline $1-5$ & $3(0.6)$ & $4(1.8)$ & $7(0.9)$ & 0.055 \\
\hline $6-12$ & $11(2.0)$ & $5(1.8)$ & $16(2.0)$ & \\
\hline $13-18$ & $152(25.6)$ & $52(23.4)$ & $204(25.1)$ & \\
\hline $19-30$ & $211(35.4)$ & $70(31.5)$ & $281(34.6)$ & \\
\hline $31-60$ & $169(29.4)$ & $78(34.5)$ & $247(30.4)$ & \\
\hline $61-90$ & $31(7.0)$ & $26(9.4)$ & $57(7.0)$ & \\
\hline Total & $577(100)$ & $235(100)$ & $812(100)$ & \\
\hline \multicolumn{5}{|l|}{ Employment status } \\
\hline Employed & $142(24.6)$ & $162(69.0)$ & $304(37.4)$ & $<0.001$ \\
\hline Self-employed & $232(40.2)$ & $27(11.5)$ & 259 (31.9) & \\
\hline Unemployed & $203(35.2)$ & $46(19.5)$ & $249(30.7)$ & \\
\hline Total & $577(100)$ & $235(100)$ & $812(100)$ & \\
\hline \multicolumn{5}{|l|}{ Treatment type } \\
\hline New case & $484(83.9)$ & $204(86.8)$ & $688(84.7)$ & 0.168 \\
\hline Transfer-in & $12(2.1)$ & $0(0.0)$ & $12(1.5)$ & \\
\hline Relapse & $30(5.2)$ & $21(8.9)$ & $51(6.3)$ & \\
\hline Treatment failure & $26(4.5)$ & $6(2.6)$ & $32(4.0)$ & \\
\hline Other & $25(4.3)$ & $4(1.7)$ & $29(3.5)$ & \\
\hline Total & $577(100)$ & $235(100)$ & $812(100)$ & \\
\hline \multicolumn{5}{|l|}{ TB infection site } \\
\hline Extra-pulmonary & $149(25.8)$ & $173(73.6)$ & $322(39.7)$ & $<0.001$ \\
\hline Pulmonary & $428(74.2)$ & $62(26.4)$ & $490(60.3)$ & \\
\hline Total & $577(100)$ & $235(100)$ & $812(100)$ & \\
\hline
\end{tabular}


Table 1: (Continued)

\begin{tabular}{|c|c|c|c|c|}
\hline \multirow[b]{2}{*}{ Characteristics } & \multicolumn{2}{|c|}{ Treatment outcome } & \multirow[b]{2}{*}{ Total (\%) } & \multirow[b]{2}{*}{$\boldsymbol{p}$-value } \\
\hline & Successful & Unsuccessful & & \\
\hline \multicolumn{5}{|l|}{ Treatment observer } \\
\hline Health worker & $252(43.7)$ & $61(26.0)$ & $313(38.6)$ & $<0.001$ \\
\hline Community health worker & $65(11.3)$ & $79(33.6)$ & $144(17.7)$ & \\
\hline Family member/friend & $260(45.0)$ & $95(40.4)$ & $355(43.7)$ & \\
\hline Total & $577(100)$ & $235(100)$ & $812(100)$ & \\
\hline \multicolumn{5}{|l|}{ HIV status } \\
\hline HIV positive & $476(82.5)$ & $198(84.2)$ & $674(83.0)$ & 1.00 \\
\hline HIV negative & $87(15.1)$ & $35(14.9)$ & $122(15.0)$ & \\
\hline Declined to be tested & $14(2.4)$ & $2(0.9)$ & $16(2.0)$ & \\
\hline Total & $577(100)$ & $235(100)$ & $812(100)$ & \\
\hline \multicolumn{5}{|l|}{ Year of diagnosis } \\
\hline 2010 & $49(8.5)$ & $19(8.1)$ & $68(8.4)$ & 0.031 \\
\hline 2011 & $84(14.6)$ & $28(11.9)$ & $112(13.8)$ & \\
\hline 2012 & $29(5.0)$ & $25(10.6)$ & $54(6.7)$ & \\
\hline 2013 & $166(28.7)$ & $87(37.0)$ & $253(31.1)$ & \\
\hline 2014 & $123(21.3)$ & $38(16.2)$ & $161(19.8)$ & \\
\hline 2015 & $126(21.9)$ & $38(16.2)$ & $164(20.2)$ & \\
\hline Total & $577(100)$ & $235(100)$ & $812(100)$ & \\
\hline \multicolumn{5}{|l|}{ Period of diagnosis } \\
\hline 2010-2012 (baseline period) & $162(28.1)$ & $72(30.6)$ & $234(28.8)$ & 0.636 \\
\hline 2013-2015 (Three l's period) & 415 (71.9) & $163(69.4)$ & $578(71.2)$ & \\
\hline Total & $577(100)$ & $235(100)$ & $812(100)$ & \\
\hline
\end{tabular}

Note: Unsuccessful treatment = treatment failure, default, death or transfer out; treatment success = a cure outcome or treatment completion.

TB, while $39.7 \%$ had extra-pulmonary TB (Table 1). Only $37.4 \%$ were employed while an additional $31.9 \%$ were self-employed.

Only 197 patients had data on occupations (see Figure 1). Notably, factory workers $(28.4 \%, n=197)$, miners/ex-miners $(23.4 \%)$, taxi drivers $(14.7 \%)$, security services personnel $(8.1 \%)$ and health workers $(4.6 \%)$ were the most predominantly known occupations.

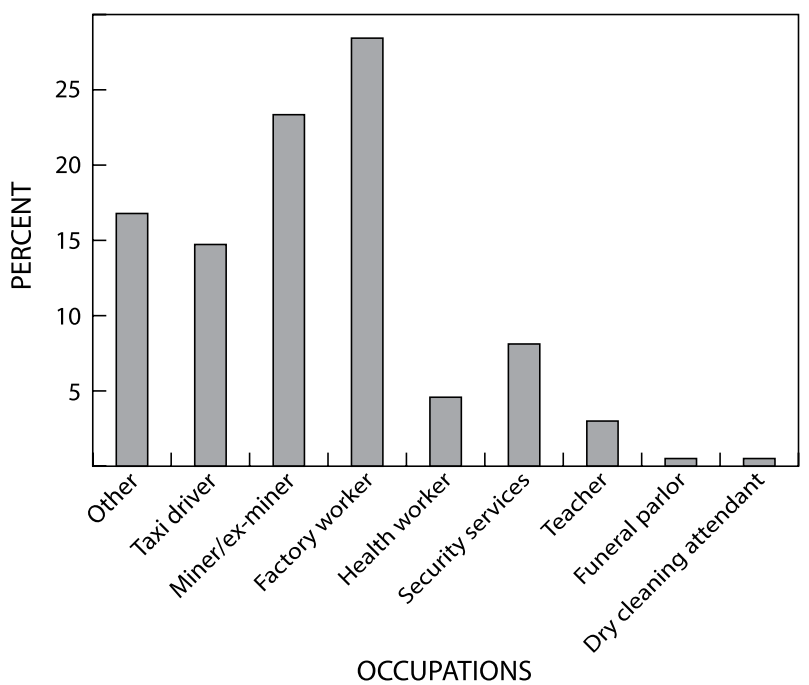

Figure 1: Percentage (\%) distribution of tuberculosis cases by occupation $(n=197)$.
Figure 2 presents the TB diagnostic techniques utilised at Senkatana HIV and TB clinic between 2010 and $2015(n=12)$. Chest X-ray was the most common diagnostic technique used from 2010 to 2015, followed by smear microscopy. The use of GeneXpert MTB/RIF increased by $17.0 \%$ between 2010 and 2012 . However, the trend in GeneXpert MTB/RIF usage dropped to 4.6\% in 2013 but then rose by $10.3 \%$ between 2013 and 2015 .

Figure 3 presents the TB treatment outcomes. Overall, 28.9\% ( $n=$ 812) of patients had unsuccessful treatment outcomes. This category with unsuccessful treatment outcomes comprised 89 $(11.0 \%, n=235)$ treatment defaults, $83(10.2 \%)$ treatment failures, $49(6.0 \%)$ deaths and $13(1.6 \%)$ transfer-out cases. Only one (0.1\%) patient had stopped treatment due to side effects.

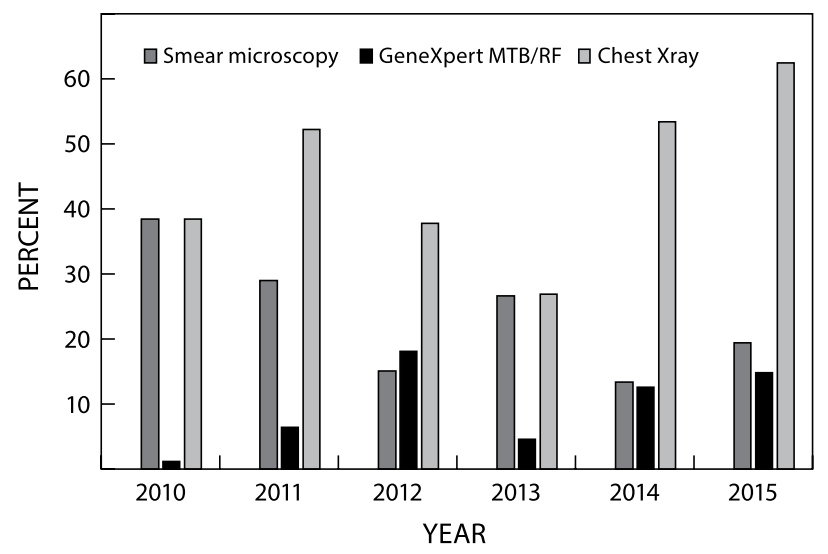

Figure 2: Diagnostic techniques used for tuberculosis at Senkatana Clinic in different years $(n=812)$. 

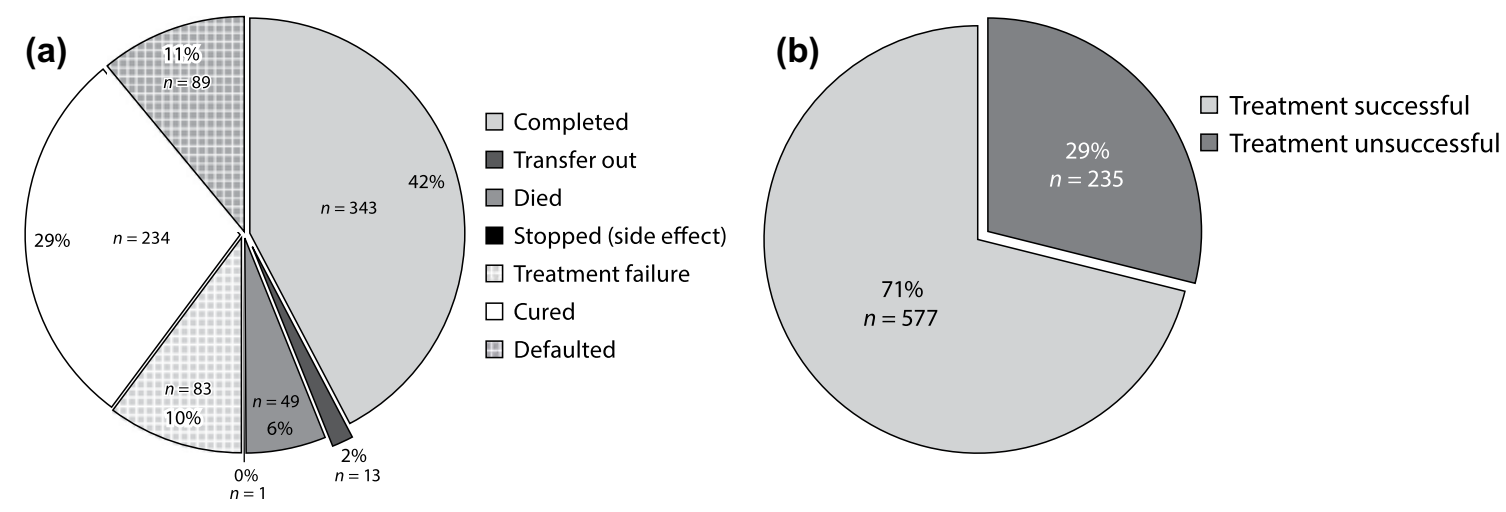

Figure 3: Tuberculosis treatment outcomes by category (A) and overall treatment outcomes (B) $(n=812)$.

Among the group with unsuccessful treatment outcomes, males had significantly $(p<0.001)$ higher $(64.2 \%, n=235)$ unsuccessful treatment outcomes compared with females (35.8\%). The 31-60 age group, with $34.5 \%(n=235)$ unsuccessful treatment outcomes, had the highest proportion of unsuccessful treatment outcomes, followed by the 19-30 age group (31.5\%). The 1-5 and $6-12$ age groups, with $0.9 \%$ and $2.0 \%$, respectively, had the lowest proportions of unsuccessful treatment outcomes. However, age group was not significantly $(p=0.055)$ associated with the treatment outcomes.

Employment status was significantly $(p<0.001)$ associated with unsuccessful treatment outcome. In total, 69.0\% $(n=235)$ of employed patients had unsuccessful treatment outcomes. About $86.8 \%$ ( $n=235)$ of new TB cases had unsuccessful TB treatment outcomes. However, treatment type was not significantly ( $p=$ 0.168 ) associated with treatment outcome (Table 1).

About $84.2 \%(n=235)$ HIV-positive patients had unsuccessful TB treatment outcomes. However, HIV status was not significantly $(p=1.000)$ associated with treatment outcome. Extra-pulmonary TB cases had a significantly $(p<0.001)$ higher proportion $(73.6 \%$, $n=235)$ of unsuccessful treatment outcomes compared with pulmonary TB (26.4\%).

Only 1.2\% ( $n=812)$ patients had records for TB microbial culture tests. Of these, $70 \%(n=10)$ had positive TB culture results. In addition, only $6.8 \%(n=812)$ had records for drug sensitivity tests (DST), inclusive of GeneXpert MTB/RIF tests (data not shown). Of these, $90.9 \%(n=55)$ and $9.1 \%$ of patients had rifampicin and isoniazid (INH) mono resistance, respectively. Further, $81.2 \%(n=812)$ patients had records indicating initiation of cotrimoxazole/dapsone for opportunistic fungal infections.

Patients supported by family members $(40.4 \%, n=235)$ had the highest proportion of unsuccessful treatment outcomes, followed by community health workers (33.6\%) and health workers $(26.0 \%)$. However, $43.7 \%(n=812)$ of the patients were observed by family members or their friends, while $38.6 \%$ ( $n=$ $812)$ were observed by health workers. Only $17.7 \%(n=812)$ of

Table 2: Logistic regression analysis of variables associated with unsuccessful TB treatment outcomes

\begin{tabular}{|c|c|c|}
\hline \multirow[b]{2}{*}{ Characteristics } & \multicolumn{2}{|c|}{ Treatment outcome } \\
\hline & Successful, $n(\%)$ & Unsuccessful, $\boldsymbol{n}$ (\%) \\
\hline \multicolumn{3}{|l|}{ Gender } \\
\hline Female & $298(51.6)$ & $150(64.2)$ \\
\hline Male & $279(48.4)$ & $85(35.8)$ \\
\hline Total & $577(100)$ & $235(100)$ \\
\hline \multicolumn{3}{|l|}{ Employment status } \\
\hline Unemployed & $142(24.6)$ & $162(69.0)$ \\
\hline Self-employed & $232(40.2)$ & $27(11.5)$ \\
\hline Employed & $203(35.2)$ & $46(19.5)$ \\
\hline Total & $577(100)$ & $235(100)$ \\
\hline \multicolumn{3}{|l|}{ TB infection site } \\
\hline Pulmonary & $428(74.2)$ & $62(26.4)$ \\
\hline Extra-pulmonary & $149(25.8)$ & $173(73.6)$ \\
\hline Total & $577(100)$ & $235(100)$ \\
\hline \multicolumn{3}{|l|}{ Treatment observer } \\
\hline Health worker & $252(43.7)$ & $61(26.0)$ \\
\hline Community health worker & $65(11.3)$ & 79 (33.6) \\
\hline Family member/friend & $260(45.0)$ & $95(40.4)$ \\
\hline Total & $577(100)$ & $235(100)$ \\
\hline
\end{tabular}

\begin{tabular}{|c|c|}
\hline Unadjusted OR (95\% CI) & $p$-value \\
\hline 1 & 0.001 \\
\hline $1.7(1.2-2.3)$ & \\
\hline 1 & \\
\hline $0.5(0.3-0.9)$ & 0.011 \\
\hline $5.0(3.4-7.4)$ & 0.001 \\
\hline 1 & 1 \\
\hline $8.0(5.6-11.3)$ & 0.001 \\
\hline 1 & \\
\hline $5.1(2.7-9.3)$ & 0.001 \\
\hline $1.5(0.9-2.6)$ & 0.138 \\
\hline
\end{tabular}

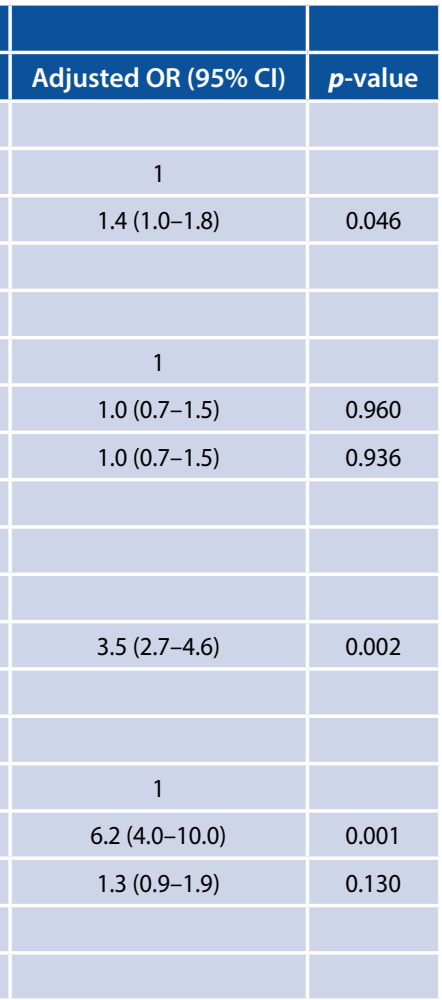


the patients were observed by community health workers. The type of treatment observer, specifically community health worker, was significantly $(p<0.001)$ associated with unsuccessful treatment outcome (see Table 1). Overall, there were no significant $(p=0.636)$ differences in TB treatment outcomes between the baseline period (2010-2012) and the period after the launch of Three I's programme (2013-2015).

Table 2 presents logistic regression analysis results of variables associated with unsuccessful TB treatment outcomes. In univariate logistic regression analysis, gender, employment status, TB infection site and treatment observer were significantly $(p<0.05)$ associated with unsuccessful treatment outcome. However, in multivariate analysis, male gender $(p=0.046)$, extra-pulmonary TB $(p=0.002)$ and community health worker $(p$ $=0.001$ ) remained significant in the final model.

\section{Discussion}

Overall, 28.9\% ( $n=812$ ) of tuberculosis (TB) patients-consisting of treatment defaults $(11.0 \%, n=235)$ cases, treatment failures (10.2\%) and deaths (6.0\%)-had unsuccessful treatment outcomes. Treatment defaults and failure of TB treatment are therefore a major concern. The fact that there was no notably significant ( $p=0.636$ ) improvement in TB treatment outcomes between the baseline period (2010-2012) and the period after the launch of Three l's programme (2013-2015) highlights the need to improve or appropriately modify the implementation of the Three I's programme. ${ }^{8}$ In particular, there is a need to improve the tracking of treatment defaulters. Harries et al. ${ }^{14}$ emphasise early tracking of initial defaulters, stating that failure to keep track of, register and report on initial defaulters is, in effect, 'sloppy' DOTS. Furthermore, the predominance of new TB cases $(84.7 \%, n=812)$ in this study population highlights the need to increase infection-control efforts. The high proportion $(83.0 \%$, 648) of HIV/TB co-infection in this population is well described by GoL. ${ }^{11}$ This highlights the need to further integrate HIV/TB intervention programmes.

Smear microscopy tests and chest X-ray were the most used diagnostic techniques in this study. The use of GeneXpert MTB/ RIF remained below 20\% $(n=812)$ between 2010 and 2015. In addition, only $1.2 \%(n=812)$ patients had records for TB microbial culture tests, while only $6.8 \%(n=812)$ of patients' files had records for drug sensitivity tests (DST). This highlights the need to scale up the use of GeneXpert MTB/RIF and DST. Ahmad and Mokaddas $^{4}$ and the $\mathrm{WHO}^{6}$ stress the need to scale up the use of the GeneXpert MTB/RIF test and drug sensitivity testing of $M$. tuberculosis strains. The fact that $90.9 \%(n=55)$ and $9.1 \%$ patients had rifampicin and isoniazid mono-resistance highlights the need to scale up drug sensitivity testing.

The proportion $(23.8 \%, n=1066)$ of patients' files without records on treatment outcomes highlights the need to improve record management. Maama-Maim, et $a l .^{15}$ corroborate the problem of poor data management in TB programmes. Tweya et al. ${ }^{16}$ note that electronic data management may lead to improved adherence to clinical guidelines and improved quality of patient care.

Male gender, employment status, extra-pulmonary site of TB infection and the use of community health workers as treatment observers proved to be important variables associated with unsuccessful treatment outcomes in univariate analysis. However, male gender (adjusted odds ratio (OR) $=1.4 ; 95 \%$ confidence interval (CI) $1.0-1.8 ; p=0.046)$, extra-pulmonary TB $(\mathrm{OR}=3.5 ; 95 \% \mathrm{Cl} 2.7-4.6 ; p=0.002)$ and community health workers as treatment observers $(\mathrm{OR}=6.2 ; 95 \% \mathrm{Cl} 4.0-10.0$; $p=0.001$ ) emerged as significant factors in multivariate analysis.

Males constituted a higher proportion $(55.2 \%, n=812)$ of the study population compared with females in this study. Floyd et al. ${ }^{17}$ corroborate this finding in Russia, where males constituted $70 \%$ ( $n=3352)$ of TB patients. Riskier occupations such as working in the mines, alcohol abuse and smoking may explain higher TB rates among men, including poor treatment outcomes. ${ }^{18-20}$ This highlights the need to scale up interventions aimed at improving diagnosis and treatment of TB in men complemented by behaviour and lifestyle change programmes.

The fact that extra-pulmonary TB emerged as a significant factor $(p=0.002)$ associated with unsuccessful treatment outcome highlights that extra-pulmonary TB is more difficult to treat in this population. Sama et al. ${ }^{21}$ highlight that there is a need for new drugs and/or dosage forms to treat extra-pulmonary TB.

TB treatment observation by community health workers is particularly less effective in this population compared with observation by other groups of observers. This may be due to inadequate staffing levels of community health workers. Interruption of treatment services during weekends and holidays may also be affecting treatment observation by health workers. This highlights the need to increase the number of community health workers and strengthen their role in monitoring TB treatment. Community health workers are an important component of the DOTS and the Three I's programmes. Therefore, their role is critical to the success of these programmes.

Although statistically insignificant in multivariate analysis, employment status was significantly $(p<0.001)$ associated with treatment outcomes in univariate analysis. Notably, factory workers (28.4\%, $n=197)$, miners/ ex-miners (23.4\%), taxi drivers (14.7\%), security services personnel (8.1\%) and health workers (4.6\%) were the most predominant occupations in this study. However, the least common occupations included teachers $(3.0 \%, n=197)$, funeral parlour attendants $(0.51 \%)$ and dry-cleaning attendants $(0.51 \%)$. There is a need to increase infection control and prevention efforts and scale up TB screening in these occupations. Stuckler et al. ${ }^{22}$ attribute higher transmission rates of TB among miners to incomplete treatment due to high rates of treatment defaults associated with migration. Further, there is a need to strengthen adherence to infection control guidelines by healthcare workers. Bhebhe, Van Rooyen and Steinberg ${ }^{23}$ note that only $22.0 \%(n=129)$ of nurses in Lesotho follow appropriate methods of sputum collection, while Mugomeri et al..$^{24}$ note that $43.6 \%(n=55)$ of the nurses have poor adherence to TB infection control guidelines.

High rates of TB infection among taxi drivers highlight the need to scale up infection control efforts in the public transport system in Maseru, Lesotho. The occurrence of tuberculosis among funeral parlour and dry-cleaning attendants is an important public health issue. Lauzardo et al. ${ }^{25}$ note that the transmission of Mycobacterium tuberculosis is highly possible during routine practice in funeral parlours.

One major limitation of this study was that data for CD4 counts, which indicate the immune status of patients, ${ }^{26}$ was not available. The immune status of the patients was therefore a potential confounding variable for some factors investigated in this study, particularly with regard to the effect of pulmonary and extrapulmonary TB on treatment outcomes of TB, as noted by Musa et al. ${ }^{27}$ Another limitation is that the study was conducted in only 
one district of Lesotho. Although Senkatana HIV and TB Clinic is the largest ART centre in Maseru District, further studies in other districts of the country are required. However, this study contributes towards the improvement of TB treatment outcomes in the country and regions worst affected by TB, particularly in countries implementing the Three l's programme of the WHO.

\section{Conclusion}

Overall, a considerable proportion of TB patients had unsuccessful treatment outcomes. The lack of significant improvement in TB treatment outcomes after the launch of the Three l's programme (2013-2015) highlights the need to improve the implementation of this programme. There is a need to scale up TB screening in high-risk groups. Male gender, extra-pulmonary TB and the use of community health workers as treatment observers are important prohibitive factors to consider in TB intervention programmes in this population. Effective drugs and/or dosage forms for improved therapeutic outcomes in extra-pulmonary TB should be considered. Furthermore, there is a need to scale up the use of rapid diagnostic techniques, particularly GeneXpert MTB/RIF.

Presentation - Part of the information in this article was presented at the 6th Infection Control Africa Network (ICAN) Congress, September 2016, Johannesburg, South Africa.

Authors' contributions - EM conceptualised the study, supervised data collection, analysed the data and drafted the manuscript with editorial inputs from BSB, CM and Prof. CT.

Acknowledgements - The authors would like to thank the hospital authorities for approving this study. They also thank Lipuo E. Mahloane, Relebohile Mautsoe and Tholoana Kamohelo for data collection.

\section{Funding - This study was self-funded by the authors.}

Disclosure statement - No potential conflict of interest was reported by the authors.

\section{ORCID}

Eltony Mugomeri (D) http://orcid.org/0000-0001-5353-7387

Bisrat S Bekele (D) http://orcid.org/0000-0001-8773-7467

Charles Maibvise iD http://orcid.org/0000-0002-1411-7491

Clemence Tarirai (D) http://orcid.org/0000-0001-5444-4457

\section{References}

1. WHO. Global tuberculosis report 2014. Geneva: World Health Organisation; 2014.

2. Khan FA, Minion J, Pai M, et al. Treatment of active tuberculosis in HIV-coinfected patients: a systematic review and meta-analysis. Clin Infect Dis. 2010;50(9):1288-99. https://doi.org/10.1086/649516

3. Perkins MD, Cunningham J. Facing the crisis: improving the diagnosis of tuberculosis in the HIV era. J Infect Dis. 2007;196(Supplement 1):S15-S27. https://doi.org/10.1086/522501

4. Ahmad S, Mokaddas E. Current status and future trends in the diagnosis and treatment of drug-susceptible and multidrugresistant tuberculosis. J Infect Public Health. 2014;7(2):75-91. https://doi.org/10.1016/j.jiph.2013.09.001

5. Palomino JC. Current developments and future perspectives for TB diagnostics. Future Microbiol. Jan 2012;7(1):59-71. https://doi.org/10.2217/fmb.11.133

6. WHO. Global tuberculosis report 2012. Geneva: World Health Organisation; 2012.

7. Kranzer K, Houben RM, Glynn JR, et al. Yield of HIV-associated tuberculosis during intensified case finding in resource-limited settings: a systematic review and meta-analysis. Lancet Infect Dis. 2010;10(2):93-102. https://doi.org/10.1016/S1473-3099(09)70326-3
8. GoL. Health sector strategic plan 2012/13-2016/17. health mo, editor. Maseru: Ministry of Health; 2013.

9. Theron G, Peter J, van Zyl-Smit R, et al. Evaluation of the Xpert MTB/ RIF assay for the diagnosis of pulmonary tuberculosis in a high HIV prevalence setting. Am J Respir Crit Care Med. 2011;184(1):132-40. https://doi.org/10.1164/rccm.201101-0056OC

10. Dhemba J, Mushonga S, Mugomeri E. Progress and challenges in eradicating extreme poverty and hunger and combating HIV/ AIDS and other diseases in Lesotho. Aworti N, Musahara H, editors. Implementation of the Millenium Development Goals: Progress and challenges in some African countries. Addis Ababa, Ethiopia: OSSERIA; 2016.

11. GoL. National TB and Leprosy Control Strategic Plan 2013-2017. Maseru: Government of Lesotho; 2013.

12. Lesotho BOS. Lesotho 2006 census of population and housing preliminary report. Maseru: Government Printers; 2007.

13. United Nations. Assessing progress in Africa toward the Millennium Development Goals. New York (NY): United Nations; 2009.

14. Harries A, Rusen I, Chiang C, et al. Registering initial defaulters and reporting on their treatment outcomes [Unresolved issues]. Int J Tuberc Lung Dis. 2009;13(7):801-3.

15. Maama-Maime LB, Mareka M, Ershova JV, et al. Antituberculosis drug resistance survey in Lesotho, 2008-2009: lessons Learned. PLoS One 2015;10(7):e0133808. https://doi.org/10.1371/journal.pone.0133808

16. Tweya $\mathrm{H}$, Feldacker C, Gadabu OJ, et al. Developing a point-of-care electronic medical record system for TB/HIV co-infected patients: experiences from Lighthouse Trust, Lilongwe, Malawi. BMC Research Notes. 2016;9(1):146. https://doi.org/10.1186/s13104-016-1943-4

17. Floyd K, Hutubessy R, Samyshkin Y, et al. Health-systems efficiency in the Russian Federation: tuberculosis control. Bull World Health Organ. 2006;84(1):43-51.

18. Negm MF, Ali TM, Elfadil SSA. Tuberculosis situation in Ismailia governorate (2002-2012) before and after direct observed therapy short course strategy (DOTS). Egypt J Chest Dis Tuberc. 2015;65(1):211-7.

19. Satti H, McLaughlin MM, Seung KJ, et al. High risk of drug-resistant tuberculosis when first-line therapy fails in a high HIV prevalence setting. Tuberculosis situation in Ismailia governorate (2002-2012) before and after Direct Observed Therapy Short Course Strategy (DOTS) Jan 2013;17(1):100-6.

20. Seung KJ, Omatayo DB, Keshavjee S, et al. Early outcomes of MDR-TB treatment in a high HIV-prevalence setting in Southern Africa. PLoS ONE 2009;4(9):e7186. https://doi.org/10.1371/journal.pone.0007186

21. Sama JN, Chida N, Polan RM, et al. High proportion of extrapulmonary tuberculosis in a low prevalence setting: a retrospective cohort study. Public Health 2016; 138:101-07. doi:10.1016/j.puhe.2016.03.033

22. Stuckler D, Basu S, McKee M, et al. Mining and risk of tuberculosis in sub-Saharan Africa. Am J Public Health 2011;101(3):524-30. https://doi.org/10.2105/AJPH.2009.175646

23. Bhebhe LT, Van Rooyen C, Steinberg WJ. Attitudes, knowledge and practices of healthcare workers regarding occupational exposure of pulmonary tuberculosis. Afr J Prim Health Care Fam Med. 2014;6(1):E1-6.

24. Mugomeri $E$, Chatanga $P$, Lefunyane $M$, et al. Adherence to tuberculosis infection control guidelines by nurses in Lesotho. Am J Infect Control. 2015;43(7):735-38. doi:10.1016/j.ajic.2015.03.016.

25. Lauzardo $M$, Lee $P$, Duncan $H$, et al. Transmission of Mycobacterium tuberculosis to a funeral director during routine embalming. Chest 2001;119(2):640-42. https://doi.org/10.1378/chest.119.2.640

26. Ackah AN, Digbeu H, Daillo K, et al. Response to treatment, mortality, and CD4 lymphocyte counts in HIV-infected persons with tuberculosis in Abidjan, Cote d'Ivoire. The Lancet. 1995;345(8950):607-10. https://doi.org/10.1016/S0140-6736(95)90519-7

27. Musa BM, Musa $B$, Muhammed $\mathrm{H}$, ]et al. Incidence of tuberculosis and immunological profile of TB/HIV co-infected patients in Nigeria. Ann Thorac Med. 2015;10(3):185-92. https://doi.org/10.4103/18171737.160838 